\title{
Tratamiento de la estenosis traqueobronquial con prótesis. Factores de buena respuesta
}

\author{
Manuel Quiroz F. ${ }^{1}$, Paulina Rojas A. ${ }^{2}$, Rafael Moya A. ${ }^{1}$, \\ Raimundo Vergara G. ${ }^{1}$, Juan Emilio Cheyre F. ${ }^{3}$, Jorge Armijo H. ${ }^{3}$, \\ Virginia Linacre S. ${ }^{3}$, Carlos Bidegain P. ${ }^{3}$ y Dimitrije Pavlov L. ${ }^{3}$
}

\section{'Hospital Clínico La Florida. Santiago, Chile ${ }^{2}$ Facultad de Medicina Universidad de los Andes. Santiago, Chile. ${ }^{3}$ Servicio de Cirugía de Tórax Instituto Nacional del Tórax. Santiago, Chile.}

Recibido el 16 de febrero de 2018 y aceptado para publicación el 24 de julio de

Correspondencia a: Dr. Manuel Quiroz F. quirozflores.manuel@gmail.

\section{Treatment of tracheobronchial stenosis with stents. Good response factors}

Aim: Determine good response predictors in use of stent in benign and malignant tracheobronchial stenosis. Materials and Methods: We retrospectively reviewed medical records of patients submitted to the procedure in the period 2014 to 2016. Clinical records, operative protocols, and histopathological and imaging studies were reviewed. Demographic and clinical data, performance status (PS) at admission and after the procedure, etiology of tracheal stenosis, need for oxygen in liters (L) Post-intervention, hospital stay, procedure-related morbidity, indication of therapy (Chemotherapy and/or Radiotherapy) and survival. Data were analyzed with descriptive and analytics statistics. Results: A total of 68 procedures were performed in 44 patients, 24 women. Tracheal stenosis 40 cases were diagnosed. The malignant etiology was $88 \%$. The admission PS was greater than or equal to 2 in $68 \%$ and $22 \%$ after the procedure. The oxygen requirements prior to the procedure were at least $1 \mathrm{~L}$ in 36 cases and decreased in 13 cases after the procedure. The average hospitalization period was 6.2 days (1-60). Complementary therapy was indicated in 13 patients; the morbidity associated with the procedure was $2.9 \%$, displaced installation and iatrogenic injury. The one year survival was $27 \%$. Conclusions: Benign etiology, el PS minor than or equal to 3 , oxygen requirements prior to the procedure of $1 \mathrm{~L}$ and tumoral obstruction less than $75 \%$ were good response predictors in our study.

Key words: airway obstruction; fistula; stents.

\section{Resumen}

Objetivo: Determinar predictores de buena respuesta con el uso de prótesis en la estenosis traqueobronquial. Materiales y Método: Estudio retrospectivo descriptivo de una serie de casos entre junio de 2014 y junio de 2016. Se revisaron registros clínicos, protocolos operatorios, estudios histopatológicos y de imagen. Se consignaron antecedentes demográficos y clínicos al ingreso y luego del procedimiento, etiología de la estenosis traqueal, necesidad de oxígeno en litros, estadía hospitalaria, morbilidad asociada al procedimiento, indicación de terapia complementaria posterior y sobrevida. Se analizaron los datos con estadística descriptiva y analítica. Resultados: Se realizaron 68 procedimientos, en 44 pacientes, 24 mujeres, el diagnóstico principal fue estenosis traqueal con 40 casos. La etiología neoplásica fue el $88 \%$ de los casos. El Performance status (PS) de ingreso fue mayor o igual a 2 en el $68 \%$ y posterior al procedimiento disminuyó a 22\% p < 0,05. En 36 casos los pacientes requerían al menos 1 L de oxígeno lo que disminuyó en 13 casos posterior a la intervención $\mathrm{p}<0,05$. El tiempo de hospitalización promedio fue 6,2 días (1-60). En 13 pacientes con patología neoplásica se indicó terapia paliativa complementaria. La morbilidad asociada al procedimiento fue de $2,9 \%$ dado por prótesis desplazada y lesión iatrogénica. La sobrevida fue de 27\% a un año. Conclusiones: La etiología benigna, el PS previo a la intervención menor o igual a 3 , requerimiento de oxígeno de 1 litro y obstrucción tumoral menor al $70 \%$ del lumen fueron elementos de buena respuesta.

Palabras clave: obstrucción de la vía aérea; fístula; prótesis. 


\section{Introducción}

Los pacientes con estenosis o fístulas de la vía aérea principal presentan un gran deterioro en su calidad de vida que está condicionada principalmente por la disnea, el estridor y la insuficiencia respiratoria ${ }^{1}$.

La etiología es principalmente neoplásica de origen broncogénico y en segundo lugar se encuentra la patología benigna. En ambos casos el uso de prótesis traqueal como tratamiento, ya sea paliativo o curativo tiene indicaciones claras y con resultados demostrados ${ }^{2}$.

La posibilidad de realizar procedimientos endoscópicos como dilatación, instalación de prótesis, sección con electrobisturí, crioterapia y braquiterapia han permitido mejorar los resultados terapéuticos en casos seleccionados.

El objetivo de este trabajo es presentar nuestra experiencia y determinar elementos predictores de buen resultado con el procedimiento.

\section{Definiciones}

Etiología neoplásica: se consideró en pacientes con biopsia de lesión primaria traqueal, invasión regional de un primario cervical bajo o mediastínico y localización secundaria de un primario conocido con biopsia confirmatoria.

Etiología benigna: se determinó mediante historia clínica, biopsia quirúrgica y estudio de laboratorio.

Tratamiento paliativo complementario: tratamiento que incluye entre sus objetivos mejorar las condiciones clínicas físicas y sicológicas del paciente cuando no es posible lograr la curación; en este trabajo se consideró radioterapia o quimioterapia posterior al procedimiento.

Indicación de procedimiento: La indicación del tratamiento en los pacientes se realizó teniendo en cuenta que el grado de estenosis de la vía aérea era mayor del $50 \%$, posibilidad de reexpansión del pulmón subyacente y relevancia de los síntomas respiratorios, fundamentalmente la disnea.

Exito del procedimiento: Se consideró mejoría de la disnea si ésta disminuía al menos un punto en la escala de la Medical Research Council. La resolución de la estenosis se definió como una repermeabilización superior al $75 \%$ de la luz traqueal.

\section{Material y Método}

Estudio retrospectivo descriptivo de una serie de casos.
Se revisaron registros clínicos, protocolos operatorios, estudios histológicos y de imágenes.

Se consignaron antecedentes demográficos y clínicos, PS al ingreso y luego del procedimiento, etiología de la estenosis traqueal, necesidad de oxígeno en litros (1) posterior a la intervención, la disnea se evaluó según la Escala Modificada de la Medical Research Council.

Se evaluó la estadía hospitalaria y morbilidad precoz y tardía asociada al procedimiento.

Registramos los pacientes que fueron a terapia complementaria posterior con intención curativa.

Evaluamos sobrevida a un año del procedimiento.

Se analizaron los datos con estadística descriptiva mediante el software SPSS ${ }^{\circledR} 11.0$ y prueba $t$ de Student y cálculo de odds ratio (OR).

\section{Procedimiento}

Se realizó en pabellón, bajo anestesia general y técnica aséptica, mediante broncoscopía rígida.

El procedimiento consistió en la identificación de la lesión en las fístulas y los extremos proximales y distales de las estenosis, los que se demarcan bajo radioscopía, se realiza toma de muestras para biopsia diferida, se determinó la necesidad de resección endoscópica del tumor o dilatación con balón en cada caso hasta lograr un lumen apropiado para desplegar la prótesis.

Se verificó la posición adecuada mediante radioscopía y visión directa. Al final del procedimiento se instila lidocaína endotraqueal. El posoperatorio se realiza en la Unidad de Cuidados Intermedios.

El tipo de prótesis se eligió por el cirujano de acuerdo a cada caso.

Se realizó radiografía de control posoperatoria inmediata.

\section{Resultados}

Se realizaron 68 procedimientos, en 44 pacientes, 24 fueron mujeres, el diagnóstico clínico más frecuente fue estenosis traqueal en 40 casos.

La etiología neoplásica fue el $88 \%$ con 39 casos (Tablas 1 y 2).

El síntoma más frecuente fue disnea en el $68 \%$ de los casos correspondiendo el $80 \%$ a disnea tipo III y el $20 \%$ a disnea tipo II, seguido por estridor en el $18 \%$ e infección respiratoria baja en el 13\%.

El PS de ingreso fue mayor o igual a 2 en el $68 \%$ de los casos, lo que se redujo al $23 \%$ luego del procedimiento $(\mathrm{p}=0,000004)$. Los pacientes que presentaban un PS de 4 tuvieron un OR de 5,5 para la variable "no mejorar" después del proce- 
Tabla 1. Características demográficas y clínicas de la población estudiada

\begin{tabular}{|llrc|}
\hline Características demográficas y clínicas & n & \% \\
Género & Masculino & 20 & $45 \%$ \\
& Femenino & 24 & $55 \%$ \\
Edad media (rango) & 65 años (28-78) & & \\
Tabaquismo & Activo & 36 & $81 \%$ \\
& Suspendido & 4 & $1 \%$ \\
Presentación clínica & Disnea & 30 & $68 \%$ \\
& Estridor & 8 & $18 \%$ \\
Diagnóstico & Infección respiratoria & 6 & $13 \%$ \\
& Estenosis traqueobronquial & 40 & $90 \%$ \\
& Estenosis y fístula* & 4 & $10 \%$ \\
\hline
\end{tabular}

*Estenosis traqueal y fístula traqueoesofágica.

dimiento comparados con los pacientes que tuvieron puntaje menor a 4 .

Los pacientes con etiología benigna presentaron mejoría de los síntomas en la totalidad de los casos.

La principal etiología neoplásica fue el cáncer pulmonar de células no pequeñas y el estadio tumoral fue igual o mayor a III en un $87 \%$ (Tablas 3 y 4 ).

La ubicación anatómica de la obstrucción se describe en la Tabla 6, la distribución de las lesiones fue homogénea, destacando el cáncer esofágico escamoso, el melanoma y el sarcoma por su presentación multifocal.

El 33\% de los pacientes disminuyó los requerimientos de oxígeno en al menos $1 \mathrm{~L}(\mathrm{p}=0,000096)$ (Tabla 2).

Los pacientes que requerían más de $1 \mathrm{~L}$ De oxígeno previo al procedimiento presentaron un OR de 3,3 para no disminuir el requerimiento posterior al procedimiento comparado con los pacientes con requerimientos de $1 \mathrm{~L}$

La prótesis que se instaló con mayor frecuencia fue el stent recto en 21 casos, seguida por la prótesis de DUMON ${ }^{\circledR}$ en Y . Como procedimiento asociado se realizaron 17 dilataciones con balón (Tabla 5).

El tiempo de hospitalización promedio fue 6,2 días (1-60), en patología maligna 13 pacientes recibieron terapia paliativa complementaria; seis pacientes recibieron quimioterapia y siete pacientes radioterapia.

La morbilidad asociada al procedimiento fue de $4 \%$, una prótesis desplazada y una lesión iatrogénica que se manifestó como un neumotórax. No presentamos mortalidad asociada al procedimiento en el período de estudio.

La sobrevida en pacientes con etiología tumoral fue de $27 \%$ a un año y la causa de muerte en todos los casos fue la progresión de la enfermedad neoplásica.

Los pacientes con etiología neoplásica y obstrucción mayor al 70\% del lumen requirieron una nueva intervención antes de
Tabla 2. Condición clínica antes y después del tratamiento

\begin{tabular}{|c|c|c|c|c|c|c|}
\hline \multirow{2}{*}{\multicolumn{2}{|c|}{$\begin{array}{l}\text { Características } \\
\text { clínicas }\end{array}$}} & \multicolumn{2}{|c|}{$\begin{array}{l}\text { Previo a la } \\
\text { intervención }\end{array}$} & \multicolumn{2}{|c|}{$\begin{array}{l}\text { Posterior a la } \\
\text { intervención }\end{array}$} & \multirow[t]{2}{*}{ Valor $p^{*}$} \\
\hline & & $\mathbf{n}$ & $\begin{array}{c}\% \\
\text { (del total) }\end{array}$ & & $\begin{array}{c}\% \\
\text { (del total) }\end{array}$ & \\
\hline \multirow{5}{*}{$\begin{array}{l}\text { Performance } \\
\text { Status }\end{array}$} & 0 & - & - & - & - & \multirow{5}{*}{0,000004} \\
\hline & 1 & 15 & $34 \%$ & 34 & $77 \%$ & \\
\hline & 2 & 8 & $18 \%$ & 3 & $7 \%$ & \\
\hline & 3 & 11 & $25 \%$ & 2 & $5 \%$ & \\
\hline & 4 & 10 & $23 \%$ & 5 & $11 \%$ & \\
\hline \multirow{3}{*}{$\begin{array}{l}\text { Necesidad } \\
\text { de oxígeno }\end{array}$} & 11 & 23 & $52 \%$ & 13 & $30 \%$ & \multirow{3}{*}{0,000096} \\
\hline & 21 & 12 & $27 \%$ & 9 & $20 \%$ & \\
\hline & 31 & 4 & $9 \%$ & 4 & $9 \%$ & \\
\hline
\end{tabular}

1: Litro. *El valor de $p$ es estadísticamente significativo para la mejoría del performance status y la disminución de los requerimientos de oxígeno posterior a la intervención.

Tabla 3. Estadio tumoral

\begin{tabular}{|ccc|}
\hline & Etiología neoplásica & \\
Estadio tumoral & $\mathbf{n}$ & $\mathbf{\%}$ \\
\hline I-II & 5 & $13 \%$ \\
\hline III-IV & 34 & $87 \%$ \\
\hline
\end{tabular}

Según el estadio tumoral para cada etiología, el $87 \%$ se encontró en una etapa avanzada de la enfermedad.

Tabla 4. Distribución según etiología

\begin{tabular}{|lcc|}
\hline Etiología & n & $\%$ \\
CPCNP & 19 & $43 \%$ \\
Cáncer esofágico escamoso & 10 & $23 \%$ \\
Metástasis & 6 & $14 \%$ \\
Granulomatosis de Wegener & 3 & $7 \%$ \\
Linfoma de células B & 2 & $4 \%$ \\
Traqueomalacia & 2 & $4 \%$ \\
Neuroendocrino pulmonar & 1 & $2 \%$ \\
Cáncer anaplástico de tiroides & 1 & $2 \%$ \\
\hline
\end{tabular}

CPCNP: Cáncer pulmonar de células no pequeñas. La etiología neoplásica fue la más frecuente, con predominio del CPCNP.

Tabla 5. Distribución según prótesis utilizada

\begin{tabular}{|cc|}
\hline Tipo de prótesis & n \\
\hline Dumon en $Y$ & 10 \\
\hline Montgomery & 10 \\
\hline Stent rectos & 21 \\
\hline Stent en $Y$ & 8 \\
\hline Stent en $\mathrm{J}$ & 3 \\
\hline
\end{tabular}

La prótesis que se utilizó con mayor frecuencia fue el stent recto considerando intervención primaria y reintervención. 
Tabla 6. Distribución según histología tumoral y localización

\begin{tabular}{|lcccc|}
\hline Histología & $\begin{array}{c}\text { Localización } \\
\text { traqueal }\end{array}$ & $\begin{array}{c}\text { Bronquio } \\
\text { derecho }\end{array}$ & $\begin{array}{c}\text { Bronquio } \\
\text { izquierdo }\end{array}$ & $\begin{array}{c}\text { Dos o más } \\
\text { localizaciones }\end{array}$ \\
CPCNP & 5 & 8 & 6 & - \\
Neuroendocrino pulmonar & 1 & - & 3 & - \\
Invasión por continuidad & & & - & - \\
$\quad$ Cáncer esofágico escamoso & 3 & - & - & - \\
$\quad$ Cáncer anaplástico de tiroides & 1 & - & - & - \\
Metástasis & & - & - \\
$\quad$ Melanoma & - & 1 & - \\
$\quad$ Células claras & 1 & 1 & - \\
Sarcoma & - & - & - & 1 \\
\hline
\end{tabular}

CPCNP: Cáncer pulmonar de células no pequeñas. Se observa una distribución homogénea, destacan el cáncer esofágico escamoso, el melanoma y el sarcoma por su localización multifocal.

dos meses con un OR 33 comparado con pacientes con obstrucción menor a $70 \%$.

\section{Discusión}

El uso de stent en la estenosis traqueobronquial es un tratamiento paliativo en pacientes que no son candidatos a cirugía, ya sea por el estadio de una enfermedad neoplásica avanzada o porque las condiciones del paciente no permiten una cirugía.

El tratamiento endoscópico se considera mínimamente invasivo y su utilidad en mejorar la calidad de vida de los pacientes está descrita ${ }^{1,2}$.

En nuestra experiencia la patología neoplásica es la principal causa de estenosis traqueobronquial que hemos tratado con prótesis, a diferencia de lo reportado por otros autores ${ }^{3-6}$, creemos que esto se debe a que somos un centro de referencia nacional con énfasis en la patología neoplásica compleja.

En el grupo de etiología benigna los diagnósticos más frecuentes de nuestra serie fueron traqueomalacia posintubación prolongada y granulomatosis de Wegener con estenosis subglótica persistente. Respecto al primer grupo, el uso de stent es una alternativa aceptada que mejora la calidad de vida en casos seleccionados, sin embargo, se debe observar de forma periódica por la posibilidad de reacciones inflamatorias y mayor riesgo de reestenosis si existe el antecedente de traqueostomía ${ }^{7}$. En el segundo grupo el rol del stent se reserva a pacientes que no han respondido al manejo médico adecuado ni a terapias de dilatación e inyecciones de esteroides por vía endoscópica ${ }^{8}$.
En el grupo de patología tumoral encontramos tumores broncogénicos en un $43 \%$, compromiso traqueal por continuidad en un $23 \%$ y metástasis en un $14 \%$, una distribución similar a la reportada en la literatura ${ }^{5,6}$. En el grupo de compromiso traqueal por continuidad se asociaron fístula y estenosis principalmente en cáncer esofágico, en estos pacientes logramos una mejoría sintomática inmediata posterior al procedimiento, lo que es compatible con lo descrito en el estudio de Madan y cols ${ }^{9}$.

Los resultados clínicos que presentamos en patología benigna fueron la mejoría en el PS y en los requerimientos de oxígeno en todos los casos, con una respuesta mantenida en los controles alejados sin necesidad de una nueva intervención.

En patología tumoral presentamos mejoría sintomática en el $65 \%$ de los casos, alcanzando una diferencia estadísticamente significativa, al igual que la disminución de requerimientos de oxígeno, sin embargo, debemos destacar que los pacientes con PS de 4 respondieron menos a la intervención, lo que concuerda con el estudio de Razi et al. ${ }^{10}$, quienes identificaron una mayor sobrevida y mejoría de los síntomas en pacientes con PS menor de 4 y disnea moderada.

En nuestra serie la instalación de stent mejoró la condición clínica en pacientes con etiología neoplásica y permitió realizar radioterapia o quimioterapia paliativa resultado en una mejoría en la calidad de vida en trece pacientes. Estudios han reportado esta posibilidad en pacientes que logran una mejoría notoria en su condición clínica luego del procedimiento ${ }^{11}$.

En relación a las reintervenciones, los pacientes 
con etiología tumoral fueron reintervenidos en un $95 \%$, principalmente, por reestenosis y la intervención se realizó antes de dos meses en todos los casos, lo que concuerda con la recomendación de realizar un control entre los dos y los cuatro meses ${ }^{5-7}$.

La morbilidad principal fue el desplazamiento de la prótesis y un neumotórax en un caso respectivamente. En ambos la complicación se detectó de forma precoz y se resolvió de forma oportuna.

El procedimiento tiene porcentajes de morbilidad variable según la serie que se analice y van desde $1,4 \%$ de morbilidad precoz hasta un $20 \%$ y desde un $20 \%$ de morbilidad tardía hasta un $70 \%$ en algunas series y con una mortalidad desde un $1 \%$ hasta un $13 \%{ }^{6-11}$.

En relación a la selección de la prótesis a utilizar, nuestro centro contaba inicialmente con alta disponibilidad de prótesis siliconada bifurcada en Y DUMON ${ }^{\circledR}$, por lo que se instalaron en primera instancia una gran cantidad, posteriormente la disponibilidad de prótesis aumentó y nos permitió tener mayor variedad para resolver diferentes situaciones, desde este punto de vista la prótesis metálica autoexpandible presentó ventajas en relación a las prótesis siliconadas, ya que permitía un mejor anclaje, disminuía el desplazamiento y su instalación resultó más fácil por el equipo quirúrgico. Por otro lado, las prótesis rectas fueron las más utilizadas y fueron de elección en lesiones traqueales únicas debido a las facilidades técnicas que presentaba su instalación, sin embargo, presentaron mayor proporción de migraciones, lo puede explicarse por su mayor uso en lesiones traqueales en tercio medio y por la progresión de la enfermedad tumoral local, este mayor desplazamiento no fue estadísticamente significativo.

\section{Conclusiones}

Respecto a factores de buena respuesta identificamos la etiología benigna como elemento de buen pronóstico en la totalidad de los casos, en pacientes con etiología neoplásica fueron elementos de buen pronóstico el PS previo a la intervención menor o igual a 3, si el requerimiento de oxígeno es 1 litro y si la obstrucción tumoral previo al procedimiento fue menor al $70 \%$ del lumen.

\section{Responsabilidades éticas}

Protección de personas y animales. Los autores declaran que para esta investigación no se han realizado experimentos en seres humanos ni en animales.

Confidencialidad de los datos. Los autores declaran que en este artículo no aparecen datos de pacientes.

Conflicto de intereses: Los autores declaran no tener conflicto de intereses.

\section{Referencias}

1. Seijo LM, Sterman DH, Seijo LM, Sterman DH. Interventional pulmonology. N Engl J Med. 2001;344:740-8.

2. Boelcskei PL, Dierkesmann R, Bauer PC, Becker HD, Bolliger CT, Wolfgang FJ. Section on respiratory endoscopy of the German Society of Pulmonology. Recommendations for bronchoscopic treatment of tracheobronchial occlusions, stenoses, and mural malignant tumors. J Bronchol. 2000;7:133-8.

3. Zhou GW, Huang HD, Sun QY, et al. Temporary placement of metallic stent could lead to long-term benefits for benign tracheobronchial stenosis. J Thorac Dis. 2015;7:398-404.

4. Chung FT, Lin SM, Chou CL, et al. Factors leading to obstructive granulation tissue formation after ultraflex stenting in benign tracheal narrowing. Thorac Cardiovasc Surg. 2010;58:102-7.

5. Cosano Povedano A, Muñoz Cabrera L, Cosano Povedano J, Rubio Sánchez N, Pascual Martínez N, Escribano Dueñas. Endoscopic treatment of central airway stenosis: five years' experience. Arch Bronconeumol 2005;41:322-7.

6. Cosano Povedano A, Muñoz Cabrera L, Cosano Povedano FJ, Rubio Sánchez J, Pascual Martínez N, Escribano Dueñas A. Endoscopic treatment of central airway stenosis: five years' experience. Arch Bronconeumol. 2005;41:322-7.

7. Ernst A, Silvestri GA, Johnstone D. Interventional pulmonary procedures: Guidelines from the American College of Chest Physicians. Chest. 2003;123:1693717.

8. Chin CS, Litle V, Yun J, Weiser T, Swanson SJ. Airway stents. Ann Thorac Surg. 2008;85:792-6.
9. Utzig MJ, Warzelhan J, Wertzel H, Berwanger I, Hasse J. Role of thoracic surgery and interventional bronchoscopy in Wegener's granulomatosis. Ann Thorac Surg. 2002;74:1948-52.

10. Madan K, Dhooria S, Sehgal IS, et al. A Multicenter Experience With the Placement of SelfExpanding Metallic Tracheobronchial Y Stents. J Bronchology Interv Pulmonol. 2016;23:29-38.

11. Razi SS, Lebovics RS, Schwartz G, et al. Timely airway stenting improves survival in patients with malignant central airway obstruction. Ann Thorac Surg. 2010;90:1088-93.

12. Marchese R, Poidomani G, Paglino G, Crimi C, Lo Nigro C, Argano V. Fully covered self-expandable metal stent in tracheobronchial disorders: clinical experience. Respiration 2015;89:49-56. 